\title{
INFLUÊNCIA DE DESPROTEINIZANTES ÁCIDOS NA QUANTIFICAÇÃO DA GLUTATIONA REDUZIDA ERITROCITÁRIA POR CLAE-UV
}

\author{
Karen L. Schott, Mariele F. Charão, Juliana Valentin, Juniara Cassol e Solange C. Garcia* \\ RS, Brasil \\ Valdeci J. Pomblum \\ Departamento de Clínica Médica, Universidade Federal de Santa Maria, Santa Maria - RS, Brasil \\ Denise Bohrer \\ Departamento de Química, Universidade Federal de Santa Maria, Santa Maria - RS, Brasil
}

Departamento de Análises Clínicas e Toxicológicas, Universidade Federal de Santa Maria, CP 5061, 97110-970 Santa Maria -

Recebido em 10/3/06; aceito em 14/6/06; publicado na web em 19/1/07

\begin{abstract}
INFLUENCE OF DEPROTEINIZING ACIDS IN ERYTHROCYTIC REDUCED GLUTATHIONE QUANTIFICATION BY HPLCUV. Large differences in reduced glutathione (GSH) levels have been found in different investigations, also in healthy people. GSH oxidation in vitro has been associated with sample acidification in the presence of oxihemoglobin. In this work, the influence of different acids on GSH determination utilizing HPLC with UV detection was evaluated. The results showed that metaphosphoric acid and sulfosalicylic acid were inadequate for analysis, because metaphosphoric acid showed to be inefficient for deproteinization and with sulfosalicylic acid loss of GSH was observed. Trichloroacetic acid did not effect GSH quantification, since the deproteinized form was immediately derivatized with 5, 5'-dithio-bis (2-nitrobenzoic) acid. Methods with TCA deproteinization presented linear results from 0.5 to $3.0 \mathrm{mM}$. The correlation coefficient between aqueous curves and GSH spiked RBC exceeded 0.99. Precision calculations showed CV lower than $10 \%$ and bias within $\pm 10 \%$ for concentrations of $0.5 ; 1.5$ and $3.0 \mathrm{mM} \mathrm{GSH}$. The recovery was higher than $94 \%$. Moreover, GSH blood concentrations were independent of hemoglobin concentrations.
\end{abstract}

Keywords: GSH; erythrocytes; deproteinizing acids.

\section{INTRODUÇÃO}

A glutationa, um tripeptídeo contendo cisteína, desempenha importantes funções em células humanas, especialmente como agente antioxidante. A forma reduzida da glutationa (GSH) mantém os grupos tióis (-SH) das proteínas, reduz ligações dissulfetos (-S-S-) induzidas pelo estresse oxidativo, neutraliza radicais livres e detoxifica eletrófilos ${ }^{1,2}$. Por isso, a concentração intracelular da GSH é um indicador da capacidade da célula em manter sua homeostase, através da neutralização de agentes oxidantes.

Na presença de peróxidos, a GSH intracelular é convertida a glutationa oxidada (GSSG) pela glutationa peroxidase, a qual catalisa a redução destes peróxidos ${ }^{3}$. A redução da GSSG a GSH é mediada pela enzima glutationa redutase, cujo substrato é o $\mathrm{NADPH}^{3}$. A glicose-6-fosfato desidrogenase, como fonte de NADPH, garante a redução da GSSG e a manutenção de uma constante da reserva de GSH, especialmente no eritrócito ${ }^{4}$. O nível de glutationa em eritrócitos está principalmente em sua forma reduzida $(99,5 \%)$ e a concentração de GSSG em eritrócitos é descrita como baixa porque esta atravessa facilmente a membrana celular ${ }^{5}$.

$\mathrm{Na}$ avaliação do estresse oxidativo, o uso de eritrócitos é aconselhável, pois durante seu ciclo vital entram em contato com as mais diversas estruturas orgânicas. Os eritrócitos perfazem $40 \%$ do volume do sangue e as espécies reativas do oxigênio que saem da célula vermelha têm potencial para causar dano a outros componentes celulares. Em contrapartida, a GSH em eritrócitos é responsável pela homeostase contra o dano oxidativo da própria célula, bem como de todas as outras células do organismo ${ }^{6}$.

Um método de referência para determinação de GSH ainda não

*e-mail: sgarpom@smail.ufsm.br foi definido ${ }^{7,8}$. No decorrer dos anos, vários métodos foram introduzidos para determinação da GSH em amostras biológicas. Métodos baseados em espectrofotometria, cromatografia líquida de alta eficiência (CLAE) e, mais recentemente, métodos baseados em eletroforese capilar e técnicas de ressonância magnética nuclear têm sido descritos ${ }^{9,10}$.

Estudo recente ${ }^{7}$ explica os fatores e mecanismos que comumente resultam na oxidação da GSH a GSSG em amostras sangüíneas e que ocorrem na fase pré-analítica. O uso de complexantes da GSH como N-etilmaleimida (NEM) ${ }^{7}$ em sangue total para prevenir a oxidação da GSH a GSSG, a hemólise e a desproteinização ácida da amostra são procedimentos pré-analíticos comuns que antecedem a quantificação da GSH no sangue total ou eritrócito ${ }^{11}$. Concentrações alteradas de GSH por artefatos têm sido associadas à acidificação da amostra na presença de oxiemoglobina ${ }^{12}$, da mesma forma que o uso de agentes complexantes da GSH, como NEM ${ }^{13,14}$, no procedimento pré-analítico. Desta forma, para o desenvolvimento de um método confiável, os procedimentos analítico e pré-analítico devem ser otimizados e validados.

É importante destacar que interferentes analíticos na quantificação de GSH também têm influência em medidas de GSSG e, conseqüentemente, na determinação da razão GSH/GSSG como índice de estresse oxidativo. É difícil avaliar com precisão o papel biológico da GSSG, uma vez que vários fatores após a coleta do sangue podem influenciar na sua concentração ${ }^{7}$. Desta forma, a determinação da GSSG e da razão GSH/GSSG em sangue humano como índices de estresse oxidativo tornam-se inexpressivos.

No presente estudo, avaliou-se a influência de diferentes ácidos e a concentração de hemoglobina na estabilidade de GSH eritrocitária, através de sua quantificação por CLAE com detector UV. Pois, um método confiável, reprodutível e exequiível para a 
quantificação de GSH eritrocitária têm importância clínicolaboratorial na avaliação da capacidade antioxidante do eritrócito, bem como para auxiliar no esclarecimento dos fenômenos fisiopatológicos e bioquímicos em patologias associadas ao estresse oxidativo.

\section{PARTE EXPERIMENTAL}

\section{Generalidades}

O equipamento de CLAE-UV consistiu de um sistema de gradiente cromatográfico Knauer modelo WellChrom, bomba quaternária, sistema organizador de solventes, misturador dinâmico, degaseificador com quatro canais, injetor manual com alça de amostragem de $20 \mu \mathrm{L}$; coluna cromatográfica Eurospher-100 C18, $5 \mu \mathrm{m}, 150 \mathrm{x}$ $4 \mathrm{~mm}$ integrada à pré-coluna $5 \times 4 \mathrm{~mm}$; detector UV Knauer; comprimento de onda $330 \mathrm{~nm}$, software Eurochrom 2000 versão 2,05 para Windows; sistema controlador de temperatura para a coluna, Haake FJ.

Utilizou-se o sistema "multipette plus" $e$ "eppendorf combitips plus" (Eppendorf, Hamburg, Germany) para dispensação do volume correto de eritrócitos nos tubos de ensaio.

Todas as soluções aquosas foram preparadas em água de Milli-Q (Millipore, Bedford, MA, USA). Os reagentes e outros produtos químicos foram obtidos de diferentes fontes. GSH, ácido 5,5'-ditio-bis (2-nitrobenzóico) (DTNB) e albumina sérica bovina, Sigma-Aldrich (St. Louis, MO, USA); ácido etilenodiaminotetracético (EDTA), ácido metafosfórico, ácido sulfossalicílico e ácido tricloroacético, Merck (Rio de Janeiro, Brasil); Azul brilhante de Coomassie, Mallinckrodt (St. Louis, USA); metanol grau HPLC, Tedia Company (Fairfield, USA); o reagente de Drabkin foi obtido da Labtest (Brasil).

Soluções estoque de padrões de GSH $20 \mathrm{mM}$ foram preparadas em solução aquosa de EDTA 0,3 mM. Soluções de trabalho, nas concentrações de 0,5 a 3,0 mM de GSH, eram preparadas diariamente a partir da solução estoque.

A participação dos doadores das amostras sangüíneas ocorreu mediante termo de consentimento e aprovação do estudo pelo Comitê de Ética do Centro de Ciências da Saúde (Registro 091/2003).

\section{Condições cromatográficas}

As condições cromatográficas adotadas foram: coluna cromatográfica de fase reversa Eurospher-100 C18, $5 \mu \mathrm{m}, 150$ x 4 mm integrada à pré-coluna Eurospher-100 C18, $5 \mu \mathrm{m}, 5$ x 4 mm, temperatura de $39{ }^{\circ} \mathrm{C}$; alça de amostragem de $20 \mu \mathrm{L}$; eluição por gradiente e detecção em $330 \mathrm{~nm}$.

A fase móvel consistiu de eluentes A e B, solução de $\mathrm{KH}_{2} \mathrm{PO}_{4} 25$ $\mathrm{mM}, \mathrm{pH} 3,83$ e metanol, respectivamente. $\mathrm{O}$ pH da solução de $\mathrm{KH}_{2} \mathrm{PO}_{4}$ foi ajustado a 3,83 com $\mathrm{H}_{3} \mathrm{PO}_{4} 4 \mathrm{M}$. A GSH foi derivatizada com DTNB. A análise foi iniciada com a injeção de $20 \mu \mathrm{L}$ do sobrenadante. O sistema por gradiente iniciou com $88 \%$ do eluente A e $12 \%$ do B, vazão de $1 \mathrm{~mL} / \mathrm{min}$. Após $3 \mathrm{~min}$, a fase móvel foi modificada para $92 \%$ de A com vazão de $0,6 \mathrm{~mL} / \mathrm{min}$. Aos $4 \mathrm{~min}$ e $31 \mathrm{~s}$ adotou-se $60 \%$ de A com vazão de $0,8 \mathrm{~mL} / \mathrm{min}$. Aos $10 \mathrm{~min} \mathrm{e}$ $31 \mathrm{~s}$ até $25 \mathrm{~min}$ retornou-se à constituição inicial, ou seja, $88 \%$ de A com vazão de $1 \mathrm{~mL} / \mathrm{min}$ para recondicionar a coluna.

\section{Amostras sangüíneas}

As amostras sanguiíneas coletadas em EDTA, $5 \mathrm{~mL}$ cada, foram obtidas por punção venosa e mantidas em banho de gelo.

Para avaliação da influência da hemoglobina na determinação da GSH, as amostras de sangue foram obtidas de indivíduos sadios
( $\mathrm{n}=20$ ), número igual de homens e mulheres, com idade entre 40 e 60 anos. Os fatores de exclusão foram tabagismo, etilismo e uso de polivitamínicos. A coleta de sangue foi realizada no Centro de Ciências da Saúde, Universidade Federal de Santa Maria, RS.

Os parâmetros sangüíneos, hematócrito $(\mathrm{Ht})$, hemoglobina $(\mathrm{Hb})$ e GSH foram determinados em duplicata. O hematócrito foi obtido por microcentrifugação, a hemoglobina foi medida através do método da cianometemoglobina utilizando o reagente de Drabkin e leitura a $540 \mathrm{~nm}$ e a GSH foi quantificada por CLAE-UV.

\section{Processamento da amostra biológica}

Separou-se uma alíquota de $1 \mathrm{~mL}$ do sangue total para medir hematócrito e hemoglobina. A outra alíquota do sangue, $4 \mathrm{~mL}$, foi utilizada para medir GSH. O processamento das amostras sangüíneas para medida de GSH, desde a coleta até a injeção no sistema cromatográfico, foi realizado a temperatura de $4{ }^{\circ} \mathrm{C}$. Imediatamente após a coleta, as amostras foram centrifugadas a $3000 \mathrm{rpm}$ por 10 min a $4{ }^{\circ} \mathrm{C}$. Os eritrócitos, $300 \mu \mathrm{L}$, foram dispensados no tubo de ensaio e receberam $125 \mu \mathrm{L}$ de solução de EDTA $0,3 \mathrm{mM}$ contendo ou não o padrão de GSH e $300 \mu \mathrm{L}$ de solução de triton x-100 20\% para hemólise. A mistura foi agitada em vortex por $1 \mathrm{~min}$.

\section{Procedimento para a desproteinização ácida}

Os ácidos metafosfórico (MPA) 5, 10, 15, 20, 30 e 50\%; sulfossalicílico (SSA) 5, 10, 15\% e tricloroacético (TCA) 5, 10, $15 \%(\mathrm{~m} / \mathrm{v})$ foram testados em duplicata para cada concentração utilizando um "pool" de eritrócitos. Foram adicionados $300 \mu \mathrm{L}$ da solução de ácido aos $725 \mu \mathrm{L}$ do hemolisado em cada tubo. Esta mistura foi agitada em vortex por $1 \mathrm{~min}$, mantida em repouso durante $20 \mathrm{~min}$ e centrifugada a $6000 \mathrm{rpm}$ por $20 \mathrm{~min}$.

\section{Avaliação da eficiência desproteinizante do ácido}

Os sobrenadantes foram observados e aqueles com aspecto não límpido foram desprezados. Nos sobrenadantes límpidos foi realizada a quantificação espectrofotométrica de proteínas, através do método de Bradford ${ }^{15}$.

\section{Avaliação da influência dos ácidos na determinação da GSH por CLAE-UV}

A perda da GSH durante a desproteinização ácida foi avaliada em três níveis de concentração 0,5; 1,0 e 1,5 mM de GSH adicionadas aos eritrócitos $(n=5)$ em duplicata. O nível basal de GSH foi paralelamente estabelecido. As áreas cromatográficas resultantes foram comparadas com as obtidas por injeção direta nas curvas de padrões aquosos de GSH (n=5) nas mesmas concentrações.

A etapa da derivatização da GSH foi realizada imediatamente após a obtenção do sobrenadante ácido. As seguintes soluções e amostras foram mantidas em banho de gelo $\left(4{ }^{\circ} \mathrm{C}\right)$ e seqüencialmente combinadas: $0,5 \mathrm{~mL}$ de tampão Tris-HCl 0,5 M, pH 8,9; $130 \mu \mathrm{L}$ de sobrenadante ácido a ser testado; $350 \mu \mathrm{L}$ de DTNB $10 \mathrm{mM}$ em $\mathrm{K}_{2} \mathrm{HPO}_{4} 0,5 \mathrm{M}$, pH 8,0 . Misturou-se sob agitação por 1 min e após 5 min de repouso a mistura foi acidificada com $100 \mu \mathrm{L}$ de $\mathrm{H}_{3} \mathrm{PO}_{4} 7 \mathrm{M}$ e centrifugada a $6000 \mathrm{rpm}$ por $10 \mathrm{~min}$. O sobrenadante foi injetado no cromatógrafo.

\section{Avaliação da influência da hemoglobina na estabilidade da GSH}

O hematócrito, a hemoglobina e a GSH foram determinados em sangue de 20 indivíduos sadios. Para determinação da GSH as áreas cromatográficas obtidas foram comparadas às da curva de calibração com adição de padrão nas concentrações 0,$5 ; 1,0 ; 1,5$; 
2,0 e 3,0 mM. Os valores de GSH, em $\mathrm{mmol} / \mathrm{L}$ eritrócitos e em $\mu \mathrm{mol} / \mathrm{g} \mathrm{Hb}$ foram comparados aos descritos na literatura.

A precisão metodológica foi realizada através de adições de GSH em amostras eritrocitárias, em três concentrações diferentes, baixa, média e alta, sendo 0,$5 ; 1,5$ e $3,0 \mathrm{mM}$, respectivamente, intra-dia e inter-dia $(\mathrm{n}=5)$.

\section{Métodos estatísticos}

Os dados foram expressos como média \pm desvio padrão e foram comparados pelo teste $t$ de Student. O coeficiente de correlação $r$ de Pearson foi usado para determinar a relação entre as concentrações de GSH e hemoglobina; o valor de $p<0,05$ foi considerado significativo.

\section{RESULTADOS E DISCUSSÃO}

A GSH é um importante antioxidante intracelular em mamíferos, $99,5 \%$ é encontrada no interior dos eritrócitos e a alteração de seus níveis pode indicar o estado antioxidante circulante. No entanto, devido à oxidação da GSH após a coleta de sangue, grandes diferenças nos níveis de GSH são encontradas em variadas investigações, inclusive em pessoas sadias. A oxidação da GSH tem sido associada à acidificação da amostra na presença de oxiemoglobina e pode resultar em formação de GSSG ou GS-SP (proteína glutationilada). Para evitar resultados artefatuais, os procedimentos pré-analítico e analítico devem ser otimizados.

\section{Considerações sobre a desproteinização ácida}

As etapas da desproteinização, bem como da hemólise são pontos críticos para manter a estabilidade da $\mathrm{GSH}^{16}$. Apesar das desvantagens descritas em alguns trabalhos sobre a utilização de ácidos para remover proteínas de amostras complexas, este procedimento é de simples execução e ainda muito utilizado, uma vez comprovada sua eficiência. A avaliação sistemática do uso do ácido desproteinizante é importante porque proteínas residuais podem interferir na análise, além de degradar o sistema cromatográfico ${ }^{17,18}$. Com o objetivo de evitar interferência com resultados artefatuais de $\mathrm{GSH}^{7,11}$ e dano ao sistema cromatográfico, avaliou-se a eficiência desproteinizante dos ácidos em várias concentrações. Observou-se que soluções contendo MPA 5 a 50\%, SSA 5 a $10 \%$ e TCA 5 a $10 \%$ utilizadas para desproteinização de eritrócitos resultaram em sobrenadantes não límpidos, insatisfatórios para as análises cromatográficas. Os resultados obtidos no presente trabalho referentes à eficiência do MPA na desproteinização podem ser associados ao estudo realizado por Stempak et al. ${ }^{17}$ que demonstraram que 1 volume de MPA 5 a $15 \%$ foi insuficiente para remover proteínas em 1 volume de amostras de sangue total. Recentemente, o efeito pró-oxidante do MPA em amostras contendo eritrócitos foi demonstrado através da quantificação de GSH e GSSG por eletroforese capilar, onde foi observada perda de GSH por oxidação a GSSG quando comparada à desproteinização realizada com o solvente orgânico acetonitrila ${ }^{19}$.

No presente trabalho, as desproteinizações realizadas com SSA $15 \%$ e TCA $15 \%$ proporcionaram sobrenadantes límpidos e concentrações residuais de proteínas $<4 \mathrm{mg} / \mathrm{L}$. Desta forma, estas soluções ácidas foram utilizadas para avaliação da estabilidade da GSH medida por CLAE-UV.

Quanto à interferência da matriz, após tratamento dos eritrócitos, hemólise seguida de desproteinização, tanto com SSA como com TCA, não se observou diferença em relação aos padrões aquosos, especialmente, no tempo de retenção da GSH, não representando, portanto, um problema analítico. E, o mesmo comportamento cromatográfico foi obtido para todas as análises, independente da presença ou não da matriz biológica. A Figura 1, mostra um cromatograma típico da quantificação de GSH em nível basal de uma amostra eritrocitária. O gradiente permitiu a separação adequada da GSH e do EDTA.

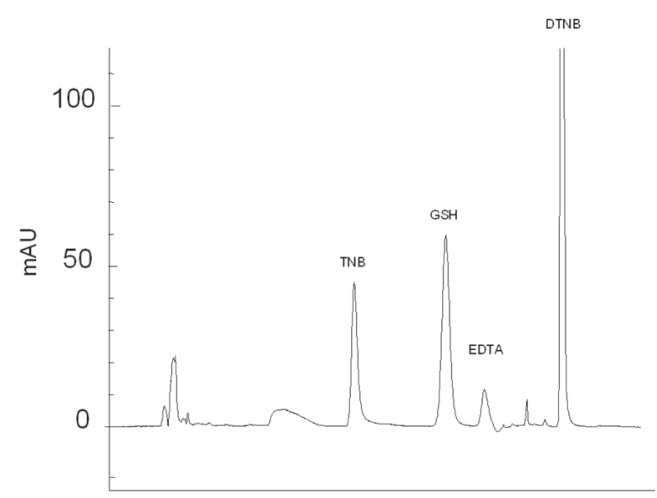

Figura 1. Um típico cromatograma do nível basal de GSH em uma amostra humana de eritrócitos após tratamento com hemólise, desproteinização com TCA e derivatização com DTNB. As condições cromatográficas foram coluna C18 (150 X $4 \mathrm{~mm}$; partículas de $5 \mu \mathrm{m}$ ); mantida a $39^{\circ} \mathrm{C}$; alça de amostragem de $20 \mu \mathrm{L}$ e detector $U V, 330 \mathrm{~nm}$; tempo total de corrida de $25 \mathrm{~min}$; fase móvel $25 \mathrm{mM} \mathrm{KH}_{2} \mathrm{PO}_{4}$ (pH 3.83):metanol; eluição por gradiente

Nos ensaios cromatográficos, a acidificação com SSA 15\% mostrou-se inadequada devido à baixa recuperação do padrão, indicando perda de GSH; para as concentrações de 0,$5 ; 1,0$ e $1,5 \mathrm{mM}$ de GSH adicionadas aos eritrócitos recuperou-se 36, 59 e $63 \%$, respectivamente. Sob as mesmas condições, o uso de TCA $15 \%$, nas três concentrações adicionadas de GSH, apresentou as recuperações de 99,5; 102,4 e 104,5\%, respectivamente. O coeficiente de variação foi menor que $10 \%$.

Desta forma, avaliou-se a estabilidade pré-analítica, utilizando TCA $15 \%$ como desproteinizante para quantificar GSH eritrocitária. A linearidade entre as curvas aquosas e as curvas com adição de padrão nas concentrações de 0,$5 ; 1,0 ; 1,5 ; 2,0$ e 3,0 mM foi realizada e os resultados mostraram que o coeficiente linear foi maior que 0,99 (Figura 2).

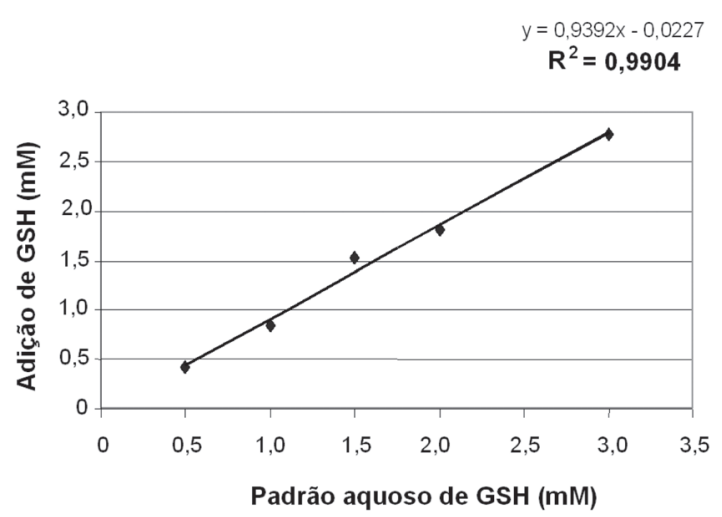

Figura 2. Representa linearidade e paralelismo entre as curvas de eritrócitos com adição de padrão $(n=5)$ e as curvas aquosas $(n=5)$ com as mesmas concentrações, sendo 0,5; 1,0; 1,5; 2,0 e 3,0 mM de GSH. A desproteinização utilizada foi com TCA $15 \%$

Para a precisão metodológica as concentrações de 0,$5 ; 1,5$ e 3,0 $\mathrm{mM}$ de GSH foram adicionadas aos eritrócitos; realizou-se, assim, a precisão intra-dia $(n=5)$ obtendo-se CV\% entre 6,64 e $9,65 \%$ e 
Tabela 1. Parâmetros sangüíneos determinados em voluntários sadios $(n=20)$. Concentrações de hemoglobina $(\mathrm{Hb})$ e hematócrito $(\mathrm{Ht})$ em sangue total e glutationa reduzida (GSH) em eritrócitos desproteinizados com TCA $15 \%$. Dados expressos em média \pm desvio padrão

\begin{tabular}{lcccc}
\hline Parâmetro & $\begin{array}{c}\mathrm{Hb} \\
(\mathrm{g} / \mathrm{dL})\end{array}$ & $\begin{array}{c}\mathrm{Ht} \\
(\%)\end{array}$ & $\begin{array}{c}\text { GSH } \\
(\mathrm{mmol} / \mathrm{l} \text { eritrócito })\end{array}$ & $\begin{array}{c}\text { GSH } \\
(\mu \mathrm{mol} / \mathrm{g} \mathrm{Hb})\end{array}$ \\
\hline Média \pm D.P. & $13,50 \pm 0,94$ & $42,40 \pm 2,41$ & $1,91 \pm 0,36$ & $5,99 \pm 0,92$
\end{tabular}

precisão inter-dia $(\mathrm{n}=5)$, para as mesmas concentrações, obtendose 7,$67 ; 4,6 ; 6,73 \%$, respectivamente. As recuperações encontradas foram de 94,$5 ; 102,4$ e $104,5 \%$ e valores de bias $-6,38 ; 1,32$ e $9,65 \%$, respectivamente. Estes resultados demonstram que o método é adequado para quantificar GSH em amostras biológicas.

A desproteinização de amostras complexas tem sido objeto de estudo no sentido de minimizar a interferência de ácidos. Carru et al. ${ }^{11}$ utilizaram membrana de filtração Microcon-10 para remover proteínas; com recuperação superior a 99,3\% da GSH adicionada aos eritrócitos, mostrando que a oxidação da GSH durante a fase pré-analítica foi minimizada.

Apesar da pesquisa de novas propostas, a desproteinização ácida continua sendo utilizada por ser um método simples e rápido. Em estudo recente ${ }^{7}$, foram descritos os fatores que causam interferência na quantificação de GSH e GSSG. Rossi et al. ${ }^{20}$ utilizaram NEM para bloquear tióis livres em amostras sangüíneas para evitar formação de dissulfetos, mas extraíram o excesso de NEM da amostra com diclorometano para evitar formação de adutos com a GSSG; após, a amostra foi desproteinizada com TCA 10\%. A partir deste procedimento, foi observada uma pequena oxidação da GSH de $\sim 3$ $4 \%$, a $0{ }^{\circ} \mathrm{C}, 20 \mathrm{~h}$ após a adição de $\mathrm{TCA}^{7}$.

A utilização de determinados ácidos para tratamento de tecidos contendo altas concentrações de grupo heme pode levar à formação de espécies oxidantes e, como conseqüência, à oxidação de grupos tióis da GSH e de proteínas, como a hemoglobina ${ }^{7}$, que constituem mais de $95 \%$ do conteúdo de proteínas no interior dos eritrócitos ${ }^{21}$. A hemoglobina humana contém seis grupos tióis por molécula, dois - SH em cada cadeia $\beta$ e $1-\mathrm{SH}$ em cada cadeia $\alpha^{22}$.

No presente estudo, os valores de hemoglobina, hematócrito e GSH estão apresentados na Tabela 1. Os valores de GSH encontrados em indivíduos sadios $(1,91 \pm 0,36 \mathrm{mmol} / \mathrm{L}$ eritrócitos $)$ são similares aos valores obtidos em recente trabalho de Luchi et al. ${ }^{23} \mathrm{e}$ com autores que utilizaram acetonitrila como agente desproteinizante $(2,05 \pm 0,42 \mathrm{mmol} / \mathrm{L} \text { eritrócitos })^{19}$.

Os resultados obtidos demonstram que o TCA não age como agente oxidante da GSH na fase pré-analítica, considerando as condições propostas neste estudo. Os níveis de GSH em eritrócitos separados do plasma imediatamente após a coleta e sem a presença de NEM, mantidos a $4{ }^{\circ} \mathrm{C}$, hemolisados com triton $\mathrm{x}-100 \mathrm{e}$ desproteinizados com TCA independem da concentração de hemoglobina total nos eritrócitos, segundo as condições metodológicas utilizadas neste trabalho, em que $p>0,05$ e coeficiente de correlação $r=-0,17$.

Rossi et al., em amplo estudo, avaliaram a influência da oxiemoglobina sobre o estado redox da glutationa e confirmaram que a glutationa total é depletada quando o sangue é acidificado com ácido perclórico e SSA. No mesmo estudo, o sobrenadante obtido com TCA mostrou pequena oxidação à GSSG, 2-3\% sob temperatura de $0{ }^{\circ} \mathrm{C}^{7}$.

Adicionalmente, a oxidação da GSH a GSSG após a coleta deve ser rigorosamente controlada, pois pode resultar, também, em falsos dados em relação a proteínas glutationiladas. De maneira geral, a formação de proteína glutationilada no eritrócito depende da formação prévia da $\mathrm{GSSG}^{23}$; assim, se houver oxidação da GSH após a coleta da amostra haverá, por consequiência indireta, a for- mação artefatual de GS-SP. Di Simplicio et al. demonstraram, em amostras de sangue tratadas com 4 volumes de TCA 5\%, que hemoglobina humana tem baixa capacidade da formar dissulfetos com a GSSG e baixa reatividade dos seus grupos -SH quando comparada com a hemoglobina de ratos $^{24}$. Desta forma, podemos reafirmar que os grupos - $\mathrm{SH}$ da hemoglobina humana, na presença de TCA, possuem baixa reatividade, o que dificultaria a perda de GSH via reação da GSSG com os grupos tióis da hemoglobina.

Ao final destas avaliações, podemos inferir que o TCA pode ser utilizado com segurança, respeitando os limites de concentração e temperatura já estudados, na desproteinização eritrocitária para determinação de GSH.

\section{CONCLUSÃO}

A acidificação eritrocitária com MPA resultou em desproteinização ineficiente e o SSA foi inadequado como agente desproteinizante, devido à baixa recuperação de GSH. Por outro lado, não foi observado efeito da concentração da $\mathrm{Hb}$ sobre a estabilidade da GSH em eritrócitos acidificados com TCA e imediatamente derivatizados com DTNB.

Os resultados encontrados demonstram que o uso de TCA $15 \%$ como agente desproteinizante nos eritrócitos é adequado, por não influenciar na estabilidade da GSH mesmo na presença de diferentes concentrações de hemoglobina. A desproteinização ácida com TCA pode ser realizada de forma rápida, o que também é importante em análises de substâncias quimicamente instáveis como a GSH.

O método utilizando TCA $15 \%$ mostrou-se adequado para determinação da GSH em eritrócitos, o que foi demonstrado através dos parâmetros acurácia (\% bias) e recuperação. Desta forma, este método pode ser utilizado com segurança para avaliação do potencial antioxidante nos eritrócitos, em estudos experimentais e clínicos envolvendo o estresse oxidativo.

\section{AGRADECIMENTOS}

Ao DAAD (Deutscher Akademischer Austauschdienst), ao Wissenschaftliche Gerätebau Dr. Ing. H. Knauer GmbH pelo suporte em cromatografia, ao CNPq e à FAPERGS pelas bolsas de estudo e suporte financeiro.

\section{REFERÊNCIAS}

1. Griffith, O. W.; Free Radical Biol. Med. 1999, 27, 922.

2. Dickinson, D. A.; Forman, H. J.; Biochem. Pharmacol. 2002, 64, 1019.

3. Comporti, M.; Chem. Phys. Lipids 1987, 45, 143.

4. Meinster, A.; Fed Proc. 1984, 43, 3031.

5. Mimic-Oka, J.; Djukanovic, L.; Markovic, B.; Biochem. Med. Met. Biol. 1998, 39, 48 .

6. Johnson, R. M.; Goyette Jr., G.; Ravindranath, Y.; Ho, Y.; Free Radical Biol. Med. 2005, 39, 1407.

7. Rossi, R.; Milzani, A.; Dalle-Done, I.; Giustarini, D.; Lusini, L.; Colombo, R.; Di Simplicio, P.; Clin. Chem. 2002, 48, 742.

8. Lada, M. W.; Kennedy, J.; Neurosci. Methods 1997, 72, 153.

9. Serru, V.; Baudin, B.; Ziegler, F.; David, J. P.; Cals, M. J.; Vaubourdolle, M.; Mario, N.; Clin. Chem. 2001, 47, 1321.

10. Reglinsky, J.; Smith, W. E.; Brzesky, M.; Marabani, M.; Sturrock, R. D.; J. Med. Chem. 1999, 35, 2134. 
11. Carru, C.; Zinellu, A.; Sotgia S.; Marongiu, G.; Farina, M. G.; Usai, M. F.; Pes, G. M.; Tadolini, B.; Deiana, L.; J. Chromatogr., A 2003, 1017, 233.

12. Gallemann Gallemann, D.; Eyer, P.; Anal. Biochem. 1990, 191, 347.

13. Eyer, P.; Podhradsky, D.; Anal. Biochem. 1996, 153, 57.

14. Beutler, E.; Srivastava, S. K.; West, C.; Biochem. Biophys. Res. Commun. 1970, 38,341 .

15. Bradford, M. M.; Anal. Biochem. 1976, 72, 248.

16. Mills, B. J.; Richie, J. P. Jr.; Lang, C. A.; Anal. Biochem. 1994, 222, 95.

17. Stempak, D.; Dallas, S.; Klein, J.; Bendayan, R.; Koren, G.; Baruchel, S.; Ther. Drug Monitor. 2001, 23, 542.

18. Lima, V. V.; Cassiano, N. M.; Cass, Q. B.; Quim. Nova. 2006, $29,72$.

19. Zinellu, A.; Sotgia S.; Usai, M. F.; Zinellu, E.; Deiana, L.; Carru, C.; Clin. Chim. Acta 2006, 366, 363.
20. Giustarini, D.; Dalle-Donne, I.; Colombo, R.; Milzani, A; Rossi, R.; Free Radical Biol. Med. 2003, 35, 1365.

21. Reishl, E. Em Non-mammalian Animal Models for Biomedical Research; Woodhead, A. W., ed.; CRC Press: Boca Raton, 1989, p. 309.

22. Amicini, G.; Brunori, M. Em Encyclopedia of Molecular Biology and Molecular Medicine; Meyers, R. A., ed.; VCH: New York, 1996, vol. 3, p. 2.

23. Lucchi, L.; Bergamini, S.; Iannone, A.; Perroni, S.; Stipo, L.; Olmeda, F.; Caruso, F.; Tomasi, A.; Albertazzi, A.; Artif. Organs 2005, 29, 67.

24. Di Simplicio, P.; Cacae, M. G.; Lusini, L.; Giannerini, F.; Giustarini, D.; Rossi, R.; Arch. Biochem. Biophys. 1998, 355, 145. 